\title{
Consideration of the Spielman-Honig Theory in the Quantitative Analysis of Coalescence Kinetics in Dilute Oil-in-Water Emulsions
}

Recent investigations $(1,2)$ in this laboratory have provided techniques for quantitatively determining particle concentration and particle size distribution changes with time in oil-in-water emulsions involving the Coulter counter. These experimental results permit for the first time a rather critical examination of the theories which attempt to describe coalescence and flocculation processes in heterodispersed systems.

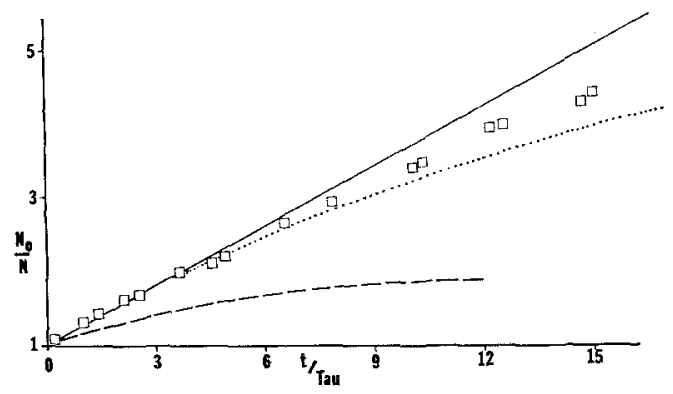

Fig. 1. Comparison of experimental data with the coalescence models with and without the correction for hydrodynamic interactions. $\square$ Experimental, $W_{\exp }=3.70 ;-$ simulation of flocculation, $W=3.70 ; \cdots$ simulation of coalescence with hydrodynamic interaction (see Ref. 7, Eq. 15 and Ref. 5 , equation 17), $\left\langle W_{i j}\right\rangle_{0}=3.69 ;$-..-- simulation of coalescence without hydrodynamic interaction (see Ref. 7, Eq. 16) $\left\langle W_{i j}\right\rangle_{0}=3.85$.

\section{Experimental}

Oil: 2:1 mixture of dioctyl and dibutyl phthalates, $0.025 \%$ by volume

Electrolyte: $0.2 \% \mathrm{NaCl}$

Surfactant: $1.25 \times 10^{-5} \%$ sodium lauryl sulfate

Temperature: $25^{\circ} \mathrm{C}$

Zeta potential: $18.1 \mathrm{mV}$ :
Computer simulation

Surface potential $=18 \mathrm{mV}$

Kappa $=0.6115 \times$ $10^{7} \mathrm{~cm}^{-1}$

$\mathrm{Hamaker}$ con$\operatorname{stan} t=1.01 \times$ $10^{-18} \mathrm{erg}$

Dielectric $=78$

Temperature $=$ $298^{\circ} \mathrm{K}$

The purpose of this report is to present some of the results of comparisons made between experiment and the coalescence theory based upon the Derjaguin - Landau - Verwey - Overbeek development $(3,4)$ with and without the recently published

Copyright (C) 1972 by Academic Press, Inc.
Spielman-Honig $(5,6)$ hydrodynamic considerations. The theoretical calculations were carried out by numerical integration (7) of the Smoluchowski kinetic equations with the Fuchs stability factor (8).

Figures 1 and 2 compare the theoretical computations with the results of an experiment using a $2: 1$ mixture of dioctyl phthalate and dibutyl

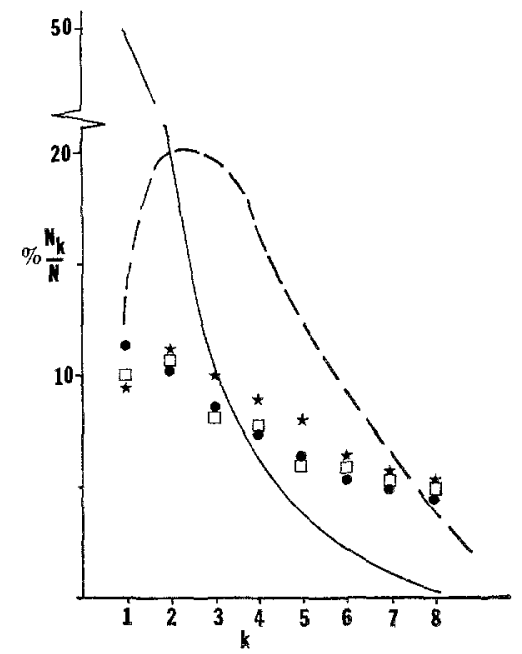

FIG. 2. Experimental particle size distribution and computer generated distributions for the flocculation model and for the coalescence model with and without the hydrodynamic effect at 8.2 Tau. -. Experimental initial distribution; $\square$ experimental distribution; 1 flocculation model distribution; $\star$ coalescence model with hydrodynamic effect, $\left\langle W_{i j}\right\rangle_{0}=3.69 ;$-..- coalescence model without hydrodynamic effect, $\left\langle W_{i j}\right\rangle_{0}=3.58$.

phthalate as the oil phase dispersed in an aqueous surfactant solution. The density of the oil mixture was matched to within $0.3 \%$ of the aqueous phase in order to permit the examination of the changes in the particle size distribution for long periods of time without the significant effects of sedimentation.

Figure 1 is a dimensionless Smoluchowski kinetic plot where $N_{0}$ is the initial total concentra- 
tion of droplets, $N$ is the total concentration of droplets at time $t$, and Tau is given by

$$
\mathrm{Tau}=3 \eta / 4 k T N_{0},
$$

where $\eta$ is the viscosity of the medium and $k T$ is the thermal energy. It can be easily seen that the theoretical calculations involving the mutual hydrodynamic interaction between particles (dotted curve) is in relatively close agreement with the experimental data while the theory which ignores this effect (dashed curve) is in poor agreement.

It is of interest to note that the theoretical calculations based upon a pure flocculation model gives the linear plot (solid line) which is also in relatively good agreement with the experimental results. However, the flocculation model should not be applicable as microscopic examinations showed that coalescence was the essential process.

In Fig. 2, the droplet size distribution at $t=$ 8.2 Tau is presented. The theoretical calculations for the two cases were carried out using the same initial experimentally determined particle size distribution (solid curve). Again, it is seen that the theory with the Spielman-Honig hydrodynamic correction is in good agreement with the experimental data. The theory without this correction gives extremely poor agreement. Comparing these results with those presented in Fig. 1, it can be seen that the particle size distribution changes with time provides a more sensitive evaluation of the two situations.

\section{ACKNOWLEDGMENT}

The support of Public Health Service Training Grant 1-F1-GM-38,054-01 is gratefully acknowledged.

\section{REFERENCES}

1. Bernstein, D. F., Higuchi, W. I., And Ho, N. F. H., J. Pharm. Sci. 60, 690 (1971).

2. Bernstein, D. F., Thesis, University of Michigan, 1971.

3. Derjagutn, B., and Landav, L., Acta Physiochim. 14, 633 (1941).

4. Verwey, E. J. W., ANd Overbenk, J. Th. G., "Theory of the Stability of Lyophobic Colloids," Elsevier, New York, 1948.

5. Spielman, L. A., J. Colloid Interface Sci. 33, $562(1970)$.

6. Honig, E. P., Wrersma, P. H., ANd Rogberson, G. J., J. Colloid Interface Sci. 36, 97 (1971).

7. Suzuki, A., Ho, N. F. H., ANd Higuchi, W. I., $J$. Colloid Interface Sci. 29, 552 (1969).

8. Kruyt, H. R., "Colloid Science," Vol. 1, Elsevier, Amsterdam, 1952.

D. F. Bernstein ${ }^{1}$

W. I. HrguchI

N. F. H. Ho

College of Pharmacy

The University of Michigan

Ann Arbor, Michigan, 48104

Received January 11, 1972; accepted January 24, $197 \%$

1 Present address, Vick Research, 1 Bradford Road, Mt. Vernon, NY 10553. 